\title{
Health-related quality of life outcomes in pediatric patients with cardiac rhythm devices: a cross-sectional study with case- control comparison
}

\author{
Helene Werner ${ }^{1,2,3^{*}}$, Phaedra Lehmann ${ }^{1,4,3}$, Alina Rüegg ${ }^{4}$, Silvia Hilfiker ${ }^{4,3}$, Karin Steinmann ${ }^{4}$ and Christian Balmer ${ }^{4,3}$
}

\begin{abstract}
Background: Little is known about health-related quality of life (HRQoL) in pediatric patients with cardiac rhythm devices. This study aims to compare self- and proxy-reported HRQoL in patients with pacemaker (PM) and implantable cardioverter-defibrillator (ICD) to that in sex- and age-matched healthy controls and to examine predictors for generic and disease-specific HRQoL.

Methods: The study included 72 PM and ICD patients (39\% females) and 72 sex- and age-matched healthy controls from 3 to 18 years of age. HRQoL data was obtained by the PedsQL 4.0 Generic Core Scales and Pediatric Cardiac Quality of Life Inventory. Medical data was collected retrospectively from medical records.

Results: Patients had significantly lower self- and proxy-reported generic overall HRQoL and lower physical health than healthy controls, and ICD patients also had lower psychosocial health. On multivariate analyses, generic overall HRQOL and physical health was significantly predicted by current cardiac medication $(\beta=-.39, p=.02$ for overall $\mathrm{HRQOL}$, respectively $\beta=-.44, p=.006$ for physical health). Disease-specific overall HRQoL was only marginally predicted by child age, device type, and the presence of a structural congenital heart disease $(p<.10)$.

Conclusions: This study shows that PM and ICD patients have lower HRQoL than healthy controls and that patients who need cardiac medication are seen by their parents at great risk for lower generic overall HRQoL. Our study also indicates a trend towards higher risk for low disease-specific HRQoL in younger patients, ICD patients, and patients with a structural congenital heart disease. Special attention should be given to these patients as they may benefit from a timely clinical evaluation in order to provide supportive interventions.
\end{abstract}

Keywords: Cardiology, Arrhythmia, Children, Adolescents, Adjustment, Adaptation, Predictors

\section{Background}

Pacemakers (PMs) and implantable cardioverterdefibrillators (ICDs) have proven to be life-saving therapeutic options for the treatment of cardiac arrhythmia. Over time, safer implant techniques and smaller devices have been introduced, and today, even very young patients can benefit from a permanent cardiac rhythm device $[1,2]$. In pediatric

\footnotetext{
* Correspondence: Helene.Werner@kispi.uzh.ch

'Department of Psychosomatics and Psychiatry, University Children's Hospital, Steinwiesstrasse 75, CH-8032 Zurich, Switzerland

${ }^{2}$ Division of Child and Adolescent Health, Institute of Psychology, University

of Zurich, Binzmühlestrasse 14, Box 8, CH-8050 Zürich, Switzerland

Full list of author information is available at the end of the article
}

patients, the most common indication for PM implantation is post-operative or congenital advanced atrioventricular block. The implantation of an ICD is indicated for primary or secondary prevention of sudden cardiac death caused by ventricular fibrillation or ventricular tachycardia, associated with congenital heart disease (CHD), genetic arrhythmia syndromes, and cardiomyopathies [2, 3]. Both interventions face various challenges such as arrhythmiarelated symptoms, post-operative complications (e.g. infections or pleural effusion), device-related complications (e.g. lead failure or battery depletion), and regular outpatient visits for clinical and device

(c) The Author(s). 2019 Open Access This article is distributed under the terms of the Creative Commons Attribution 4.0 International License (http://creativecommons.org/licenses/by/4.0/), which permits unrestricted use, distribution, and reproduction in any medium, provided you give appropriate credit to the original author(s) and the source, provide a link to the Creative Commons license, and indicate if changes were made. The Creative Commons Public Domain Dedication waiver (http://creativecommons.org/publicdomain/zero/1.0/) applies to the data made available in this article, unless otherwise stated. 
examination. In fact, pediatric patients with PM or ICD deal with life-long dependence on medical assistance and must cope with restrictions in daily activities and reduced life expectancy $[4,5]$. This can be very stressful and negatively impact their healthrelated quality of life (HRQoL).

HRQoL is a multidimensional concept that evaluates the subjective perception of the impact of health status on physical, psychological, and social functioning and wellbeing [6]. Knowledge of HRQoL is important and can help to identify subjects who are at risk for health problems and need professional assistance. Thus, it can be used to adequately support patients in their adjustment to chronic disease. Previously, few studies have described both generic and disease-specific HRQoL in pediatric patients with PMs, with ICDs, or both [7-9]. The majority of studies that used a generic HRQoL instrument indicated that patients with PMs or ICDs or both have lower HRQoL levels than healthy controls [7-14]. However, medical risk factors for impaired HRQoL have not been studied systematically. One of these studies with PM and ICD patients identified the presence of $\mathrm{CHD}$ and ICD as key drivers of lower HRQoL [7]. Two studies found no association between generic HRQoL and cardiac illness severity in ICD patients $[8,12]$, and one other study found left ventricular ejection fraction lower than $55 \%$ and intake of cardiovascular drugs associated with lower generic HRQoL [15].

The aims of this study were threefold. Firstly, we wanted to compare self- and proxy-reported generic HRQoL outcomes in PM and ICD patients to those in sex- and age-matched healthy controls. Lower HRQoL was expected in patients than in healthy controls. Secondly, we wanted to describe the level of self- and proxy-reported disease-specific HRQoL in PM and ICD patients: We expected that ICD patients would show lower HRQoL than PM patients. Thirdly, we aimed to examine associations between patients' socio-demographic and medical characteristics and proxy-reported generic and disease-specific HRQoL. Based on previous results [7], we expected to identify lower HRQoL in ICD patients and in patients with structural CHD.

\section{Methods}

\section{Participants}

This comparative cross-sectional study includes pediatric cardiac rhythm device patients and sex- and agematched healthy controls from 3 to 18 years of age. All PM and ICD patients, with or without complaints, scheduled for regular follow-up visits at the cardiology outpatient clinic of the University Children's Hospital Zurich between September 2015 and September 2016 were asked to participate in the study. Patients with PM or ICD implantation within the last 3 months prior to study were excluded to minimize the influence of acute medical effects on HRQoL. Further exclusion criteria were permanent residency outside Switzerland, lack of German language fluency in patient or caregiver, refusal to sign the informed consent, the presence of Down syndrome, and severe mental retardation. The healthy controls were matched in age and sex and recruited via best friends of the patients (40\%), and advertisements placed at the University of Zurich and community day care centers (60\%). Interviews and standardized questionnaires ensured that the controls had no chronic disease or cognitive impairments. The study was approved by the Ethical Review Board of the Canton of Zurich, Switzerland, and was performed in full accordance with the Declaration of Helsinki. All parents provided written informed consent after the study procedures and aims had been explained in detail. All pediatric patients provided verbal assent, while adolescents older than 14 years of age also provided written informed consent.

In total, 90 pediatric patients with PM or ICD were eligible for study inclusion. Of these, 72 patients were included (response rate 80\%). Eighteen patients did not participate, for the following reasons: lack of time or interest $(n=13)$, lost to follow-up $(n=4)$, and twins $(n=1$; both twins took part in the study, but only 1 child was randomly selected for inclusion in the analysis, because parent-reported data on HRQoL are correlated in such cases). Study participants did not significantly differ from the 18 non-participants in their sex $\left(\chi^{2}=.19, p=.79\right)$, age at study beginning $(\mathrm{U}=-.98, p=.33)$, device type $\left(\mathrm{X}^{2}=.02, p=.99\right)$, age at initial device implantation $(\mathrm{U}=-.93, p=.35)$, presence of structural CHD $\left(\chi^{2}=.18, p=.79\right)$, or total length of cardiac hospitalization $(\mathrm{U}=-.80, p=.43)$.

\section{Measurements}

The time since initial device implantation (years) was calculated by the difference between the child's age at HRQoL assessment and age at initial device implantation. ICD patients were grouped by whether they had experienced an aborted cardiac arrest or life-threatening arrhythmia (secondary prevention) or not (primary prevention) [16]. The severity of the disease in ICD patients was defined by using the ICD severity index [12]. Patients with structural CHDs were categorized by the complexity of the surgical repair into univentricular (i.e. Fontan type) and biventricular physiology. Previous open-heart surgery was dichotomized into yes or no. The number of device-related post-initial-implant surgeries was categorized into none, one, or more than one. The length of each hospital stay related to cardiac disease was summed into the total length of cardiac hospitalization (days). Patients' current cardiac medication was assessed by the intake of heart failure and/or 
antiarrhythmic medication, dichotomized into yes or no. In addition, the presence of any other, non-cardiac chronic disease (e.g. neurological diseases with recurrent seizures) was noted.

Generic HRQoL was assessed using the validated and authorized German version of the Pediatric Quality of Life (PedsQL) 4.0 Generic Core Scales [17]. It is a widely used 23-item measure for assessing HRQoL during the past month by proxy-reports in the age range of 2 to 18 years and by child self-report in the age range of 5 to 18 years. This instrument encompasses the following 4 subscales: physical functioning (8 items), social functioning (5 items), emotional functioning (5 items), and school functioning (5 items). The physical health summary score is defined by the physical functioning subscale. The psychosocial health summary score is computed as the sum of the items over the number of items answered in the emotional, social and school functioning subscales. In addition, a total score (generic overall HRQoL) can be computed as the sum of all the items divided by the number of items answered on all subscales. Items are reverse scored and linearly transformed to a $0-100$ scale, with higher scores indicating better HRQoL.

Disease-specific HRQoL was assessed using an authorized German version of the standardized Pediatric Cardiac Quality of Life Inventory (PCQLI), which was supplemented by a preschool instrument $[18,19]$. Thus, we used the PCQLI consisting of two self-report versions (for ages $8-12$ and 13-18) and three proxy-report versions (for ages $3-7,8-12$, and 13-18). Both self- and proxy-reported versions include 27 items (ages 8-12) and 38 items (ages 1318) comprising three subscales: 'impact of disease', 'psychosocial impact', and 'emotional environment'. A total score for disease-specific overall HRQoL is calculated by the sum of the 'impact of disease' and 'psychosocial impact' subscales. The proxy-report version for preschool children (ages 3-8) includes 52 items. A total score, computed by the sum of all items, defines the overall disease-specific HRQoL. All scales were linearly transformed into a $0-100$ scale, with higher scores indicating better HRQoL. Cut-off scores for very low, low, and normal disease-specific HRQoL total scores are available calculated from a German sample of 546 children with heart diseases [20].

The occurrence of major life events in the family during the 12 months prior to assessment was assessed by parental report, using a list of the following 12 life events: birth of a child, divorce, marriage, person moving into the household, significant change in family income, indebtedness, relocation, job change of either parent, unemployment of either parent, serious illness or accident of a family member, death of family member or a close friend, and child's change of school [21]. A life event score was computed by summing the number of life events (range $0-12$ ).
Child nationality and socioeconomic status (SES) were assessed by a short parent-reported socio-demographic questionnaire. SES was assessed by maternal education and paternal occupation on a scale from 2 to 12 , with 2 being the lowest and 12 the highest SES score. Three social classes were defined: lower (SES 2 to 5), middle (SES 6 to 9), and upper class (SES 10 to 12). This measure has proven to be a valid indicator of SES in previous studies involving the Swiss population [22].

\section{Study procedure}

After informed consent had been provided, HRQoL data was obtained using standardized questionnaires. To assess proxy-reports of child HRQoL from 3 to 18 years, questionnaires were mailed to participating parents, who completed them at home. Because valid and reliable selfreported HRQoL data can be assessed from 7 to 8 years of age [23], no self-reports were assessed for patients younger than 7.5 years. For older patients, self-reported HRQoL data was obtained in face-to-face interviews that strictly followed the procedure in the questionnaire. To ensure that the patients could express their own views openly, they were interviewed separately from their parents. The interviews were conducted by the first, second, or third author, all of whom had been trained. Self- and proxy-reported HRQoL data was assessed within 2 weeks. Patients' medical data was collected retrospectively from medical records.

\section{Statistical analyses}

Data were analyzed using the SPSS statistical package, release 22.0 for Windows (SPSS Inc., Chicago, IL, USA). All statistical tests were two-sided with a predefined significance level of $p<.05$. Chi-square tests and MannWhitney U-tests were used, as appropriate, to compare child sex, age at study beginning, device type, age at device implantation, the presence of structural $\mathrm{CHD}$ and the total length of cardiac hospitalization between study participants and non-participants. The differences in generic HRQoL between patient sample and control group and between self- and proxy-reports were analyzed by testing first the interaction between study group (patient vs. control) and report (self vs. proxy), and then the differences between patient and control group on one side and between self- and proxy-reports on the other side (see caption of Table 2). Associations between medical variables and generic and disease-specific HRQoL scores (self- and proxy-reports) were measured by non-parametric Kendall's rank correlation coefficients. Because some medical variables were continuous while others were categorical or dichotomous, we decided to use this coefficient in all cases. Four separate multiple linear regression models with identical variables were used to predict HRQoL (with the generic overall 
HRQoL score, the physical health and psychosocial health summary score, and the disease-specific overall HRQoL score as dependent variables). Selection of predictors was based on a priori hypotheses and on the statistical significance of bivariate correlations with the total score. Eight predictors were entered: child sex, age at assessment (years), time since initial device implantation (years), device type, structural CHD, current cardiac medication, total length of cardiac hospitalization (days), and other non-cardiac chronic disease. The absence of multi-collinearity was confirmed by examining the correlation matrix (correlations $>.80$ ) and the variance inflation factor [24].

\section{Results}

Patient socio-demographic and medical characteristics for the total sample and the two device types are summarized in Table 1 . The majority of the patients $(82 \%)$ had a PM device, while 13 patients (18\%) had an ICD. Patients with an ICD were significantly older at assessment and initial device implantation than patients with PM. Four of the $59 \mathrm{PM}$ patients (7\%) and 5 of the 13 ICD patients $(39 \%)$ had experienced resuscitation before implantation. Overall, 40 of the 72 PM and ICD patients (56\%) had a CHD, and 33 of these $(83 \%)$ had also undergone open-heart surgery. The time since last devicerelated surgery was significantly shorter in ICD patients than in PM patients, andICD patients needed more antiarrhythmic medication than PM patients. In total, drug therapy for heart failure or arrhythmia was present in 23 of 72 patients (32.9\%), with 3 patients (4.2\%) who needed both medications, 14 patients $(19.4 \%)$ antiarrhythmic medication only, and 3 patients (4.2\%) heart failure medication only. An additional non-cardiac chronic disease was present in 13 of 72 patients (18\%): attention deficit hyperactivity disorder $(n=3)$, hearing disorder $(n=1)$, congenital hand or arm malformation $(n=2)$, diabetes mellitus $(n=1)$, neurological disease $(n=5)$, and diseases of the endocrine system $(n=1)$.

The sex- and age-matched healthy controls did not differ significantly from the patient sample in Swiss nationality (patient group: $82 \%$; control group: $83 \%$ ). In contrast, controls had significantly higher SES: 33 of the 72 controls (46\%) belonged to the upper SES class, but only 17 of the 72 patients $(24 \%)$ did. Furthermore, parents of patients reported having experienced more previous life events than parents of controls $(p<.001$; patient group: $\mathrm{M}=2.2, \mathrm{SD}=1.9$, range $=0-7$; control group: $\mathrm{M}=0.8, \mathrm{SD}=1.0$, range $=0-4)$. In both samples, death of a family member or a close friend (patient sample: $39 \%$, control group: 18\%) and a job change of either parent (patient sample: $34 \%$, control group: $21 \%$ ) were the most frequently reported life events.
Mean values of self- and proxy-reported generic HRQoL scores for the patient sample and control group are presented in Table 2 separately for the PM and ICD groups. None of the interactions between study group (patient vs. control group) and report (self vs. proxy) was significant. This means that average HRQoL differences between patients and controls are the same for self- and proxy-reported HRQoL data. Our analyses showed that parents of PM and ICD patients reported significantly lower overall HRQoL and lower physical health summary scores than did parents of healthy controls. In addition, parents of ICD patients reported lower psychosocial health summary scores. No significant differences were found between self- and proxy-reported generic HRQoL scores.

The number of patients with very low, low, and normal disease-specific HRQoL (proxy- and self-reported data) are indicated in Fig. 1. The majority of the patients have normal proxy- and self-reported disease-specific HRQoL. Chi-square tests revealed that ICD patients more frequently had very low proxy-reported diseasespecific HRQoL than did PM patients $\left(\chi^{2}=10.7\right.$, $p=.005)$. No such difference was found in the selfreported data between PM and ICD patients $\left(\chi^{2}=1.01\right.$, $p=.60$ ). Among ICD patients, $25 \%$ of the parents reported very low disease-specific HRQoL for their child, while no patient reported very low and $9 \%$ reported low disease-specific HRQoL. Thus, significant differences between self- and proxy-reported disease-specific HRQoL scores were found in all subscales and the total score $(p=.002, d=-.47)$.

Associations between generic or disease-specific HRQoL scores and socio-demographic and medical characteristics are presented in Table 3. Kendall correlation coefficients showed that generic HRQoL scores were not significantly associated with patients' socio-demographic characteristics, while it was associated with current cardiac medication. Disease-specific HRQoL scores were significantly associated with child age at assessment, the presence of a structural $\mathrm{CHD}$ and a longer total length of cardiac hospitalization.

Table 4 summarizes the results of four separate multiple regression analyses predicting proxy-reported generic and disease-specific HRQoL (generic overall HRQoL score, physical health and psychosocial health summary, and disease-specific overall HRQoL). In regression models predicting generic HRQoL, child sex and age were not significant predictors. Among the medical variables, current cardiac medication was a significant predictor for overall HRQoL and the physical health summary score, while no significant association was found with the psychosocial health summary score. The need for cardiac medication was associated with lower generic overall HRQoL and physical health. In addition, 
Table 1 Patient socio-demographic and medical characteristics by cardiac rhythm device type

\begin{tabular}{|c|c|c|c|c|}
\hline & $\begin{array}{l}\text { Total sample } \\
(n=72)\end{array}$ & $\begin{array}{l}\mathrm{PM} \\
(n=59)\end{array}$ & $\begin{array}{l}\mathrm{ICD} \\
(n=13)\end{array}$ & $\begin{array}{l}\text { Comparison: } \\
\text { PM vs. ICD group } \\
p \text { value }^{\dagger}\end{array}$ \\
\hline Female sex, n (\%) & $28(39)$ & $25(42)$ & $3(23)$ & .23 \\
\hline Age at assessment (years), mean (SD), range & $11.3(4.6), 3.2-18.0$ & $10.5(4.4), 3.2-17.9$ & 14.9 (3.2), 7.6-18.0 & $<.001$ \\
\hline Swiss nationality, n (\%) & $59(82)$ & $47(80)$ & $12(92)$ & .44 \\
\hline Socio-economic status, n (\%) & & & & .23 \\
\hline Lower class & $3(4)$ & $3(6)$ & 0 & \\
\hline Middle class & $49(68)$ & $38(64)$ & $11(84)$ & \\
\hline Upper class & $17(24)$ & $16(27)$ & $1(8)$ & \\
\hline Unknown & $3(4)$ & $2(3)$ & $1(8)$ & \\
\hline Number of previous life events, mean (SD), range & $2.2(1.9), 0-7$ & $2.3(2.0), 0-7$ & $1.7(1.3), 0-4$ & .44 \\
\hline Age at initial device implant (years), mean (SD), range & $4.7(4.4), 0-15.3$ & $3.5(3.5), 0-12.6$ & $9.8(4.0), 2.9-15.3$ & $<.001$ \\
\hline Newborn (age $\leq 28$ days), n (\%) & $7(10)$ & $7(12)$ & 0 & \\
\hline Infant/toddler (29 days to 3 years), n (\%) & $23(32)$ & $22(37)$ & $1(8)$ & \\
\hline Preschool (3 to 6 years), n (\%) & $18(25)$ & $17(29)$ & $1(8)$ & \\
\hline School-age (6 to 13 years), n (\%) & $21(29)$ & $13(22)$ & $8(62)$ & \\
\hline Adolescent (age > 13 years), n (\%) & $3(4)$ & 0 & $3(23)$ & \\
\hline $\begin{array}{l}\text { Time since initial device implant (years), mean } \\
\text { (SD), range }\end{array}$ & $6.7(4.5), 0.4-17.9$ & $7.1(4.6), 0.4-17.9$ & $5.1(3.9), 0.5-12.7$ & .23 \\
\hline \multicolumn{5}{|l|}{ Device system, n (\%) } \\
\hline Single-chamber & $9(13)$ & $4(7)$ & $5(39)$ & \\
\hline Dual-chamber & $63(87)$ & $55(93)$ & $8(61)$ & .002 \\
\hline \multicolumn{5}{|l|}{ Location of electrode, n (\%) } \\
\hline Epicardial & $69(96)$ & $59(100)$ & $10(77)$ & \\
\hline Endocardial & $3(4)$ & 0 & $3(23)$ & NA \\
\hline \multicolumn{5}{|l|}{ Electrophysiological disease, n (\%) } \\
\hline Postoperative heart block & $20(28)$ & $20(34)$ & & \\
\hline Congenital heart block & $25(35)$ & $25(42)$ & & \\
\hline Sinoatrial node disease & $14(19)$ & $14(24)$ & & \\
\hline Ventricular tachycardia or fibrillation ${ }^{a}$ & $13(18)$ & & $13(100)$ & NA \\
\hline \multicolumn{5}{|l|}{ ICD indication, n (\%) } \\
\hline Primary prevention & NA & NA & $7(54)$ & \\
\hline Secondary prevention & NA & NA & $6(46)$ & NA \\
\hline ICD Severity Index, mean (SD), range & NA & NA & $8.0(3.8), 3-16$ & NA \\
\hline \multicolumn{5}{|l|}{ Structural congenital heart disease, $\mathrm{n}(\%)$} \\
\hline Biventricular physiology & $34(47)$ & $26(44)$ & $8(61)$ & \\
\hline Univentricular physiology & $6(8)$ & $6(10)$ & 0 & \\
\hline No structural congenital heart disease & $32(45)$ & $27(46)$ & $5(39)$ & .34 \\
\hline Previous open heart surgery, n (\%) & $33(46)$ & $30(51)$ & $3(23)$ & .07 \\
\hline \multicolumn{5}{|l|}{$\begin{array}{l}\text { Number of device-related post-initial-implant } \\
\text { surgeries, n (\%) }\end{array}$} \\
\hline None & $38(53)$ & $33(56)$ & $5(39)$ & \\
\hline One & $21(29)$ & $15(25)$ & $6(46)$ & \\
\hline More than one & $13(18)$ & $11(19)$ & $2(15)$ & .25 \\
\hline $\begin{array}{l}\text { Time since last device-related surgery (years), } \\
\text { mean (SD), range }\end{array}$ & $3.5(2.6), 0.3-12.7$ & $3.9(2.6), 0.3-12.7$ & $1.8(1.5), 0.3-5.4$ & .004 \\
\hline
\end{tabular}


Table 1 Patient socio-demographic and medical characteristics by cardiac rhythm device type (Continued)

\begin{tabular}{|c|c|c|c|c|}
\hline & $\begin{array}{l}\text { Total sample } \\
(n=72)\end{array}$ & $\begin{array}{l}\mathrm{PM} \\
(n=59)\end{array}$ & $\begin{array}{l}\mathrm{ICD} \\
(n=13)\end{array}$ & $\begin{array}{l}\text { Comparison: } \\
\text { PM vs. ICD group } \\
p \text { value }^{\dagger}\end{array}$ \\
\hline $\begin{array}{l}\text { Total length of cardiac hospitalization (days), } \\
\text { mean (SD), range }\end{array}$ & $52.4(32.5), 2-266$ & $57.0(62.4), 2-266$ & $31.5(33.0), 3-133$ & .26 \\
\hline \multicolumn{5}{|l|}{ Current cardiac medication } \\
\hline Antiarrhythmic, n (\%) & $17(24)$ & $7(12)$ & $10(77)$ & $<.001$ \\
\hline Heart failure, n (\%) & $6(8)$ & $5(9)$ & $1(8)$ & .93 \\
\hline Other non-cardiac chronic disease, n (\%) & $13(18)$ & $9(15)$ & $4(31)$ & .19 \\
\hline
\end{tabular}

NA Not applicable, PM Pacemaker, ICD Implantable cardioverter-defibrillator

${ }^{\dagger}$ Chi square tests or Mann-Whitney-U tests were performed

a Patients with documented ventricular tachycardia or fibrillation or with a significant risk of ventricular tachycardia or fibrillation Significant values are indicated in bold

Table 2 Sample means for generic health-related quality of life in pediatric patients with PM/ICD and their controls: comparison between patients and control group and between self- and proxy report

\begin{tabular}{|c|c|c|c|c|c|c|c|c|c|c|c|c|c|c|c|}
\hline & \multicolumn{5}{|c|}{ Parent proxy-report form } & \multicolumn{5}{|c|}{ Child self-report form } & \multirow{3}{*}{$\begin{array}{l}\text { Interaction: } \\
\text { Study } \\
\text { group } \\
\text { (patient/ } \\
\text { control) vs. } \\
\text { report } \\
\text { (self/proxy) } \\
\text { p-value }^{+}\end{array}$} & \multirow{2}{*}{\multicolumn{2}{|c|}{$\begin{array}{l}\text { Differences } \\
\text { Patient sample } \\
\text { vs. control } \\
\text { group } \\
\text { (all control - } \\
\text { patient } \\
\text { differences) }\end{array}$}} & \multirow{2}{*}{\multicolumn{2}{|c|}{$\begin{array}{l}\text { Differences } \\
\text { Self- vs. proxy } \\
\text { report } \\
\text { (all self - proxy } \\
\text { differences) }\end{array}$}} \\
\hline & \multicolumn{3}{|c|}{ Patient sample } & \multicolumn{2}{|c|}{$\begin{array}{l}\text { Control } \\
\text { group }\end{array}$} & \multicolumn{3}{|c|}{ Patient sample } & \multicolumn{2}{|c|}{$\begin{array}{l}\text { Control } \\
\text { group }\end{array}$} & & & & & \\
\hline & $\begin{array}{l}\text { Device } \\
\text { type }\end{array}$ & $n$ & $\mathrm{M}(\mathrm{SD})$ & $n$ & $\mathrm{M}(\mathrm{SD})$ & $\begin{array}{l}\text { Device } \\
\text { type }\end{array}$ & $n$ & $\mathrm{M}(\mathrm{SD})$ & $\mathrm{n}$ & M (SD) & & $\mathrm{M}(\mathrm{SD})$ & $\begin{array}{l}p^{-} \\
\text {value }\end{array}$ & $M(S D)$ & $\begin{array}{l}p- \\
\text { value }\end{array}$ \\
\hline \multicolumn{16}{|l|}{ PedsQL scales } \\
\hline Total Score & PM & 57 & $\begin{array}{l}83.0 \\
(14.5)\end{array}$ & 59 & $\begin{array}{l}89.9 \\
(8.1)\end{array}$ & PM & 39 & $\begin{array}{l}82.1 \\
(11.6)\end{array}$ & 42 & $\begin{array}{l}86.7 \\
(12.2)\end{array}$ & .34 & $\begin{array}{l}7.1 \\
(17.8)\end{array}$ & .007 & $\begin{array}{l}-1.3 \\
(9.8)\end{array}$ & .21 \\
\hline $\begin{array}{l}\text { Physical Health } \\
\text { Summary }\end{array}$ & PM & 57 & $\begin{array}{l}86.4 \\
(16.5)\end{array}$ & 59 & $\begin{array}{l}94.6 \\
(8.5)\end{array}$ & PM & 39 & $\begin{array}{l}84.0 \\
(13.9)\end{array}$ & 42 & $\begin{array}{l}87.6 \\
(14.2)\end{array}$ & .24 & $\begin{array}{l}8.7 \\
(18.4)\end{array}$ & $\leq .001$ & $\begin{array}{l}-2.8 \\
(12.6)\end{array}$ & .06 \\
\hline $\begin{array}{l}\text { Psychosocial Health } \\
\text { Summary }\end{array}$ & PM & 57 & $\begin{array}{l}79.6 \\
(14.3)\end{array}$ & 59 & $\begin{array}{l}85.1 \\
(9.6)\end{array}$ & PM & 39 & $\begin{array}{l}80.3 \\
(11.6)\end{array}$ & 42 & $\begin{array}{l}85.8 \\
(11.9)\end{array}$ & .85 & $\begin{array}{l}5.4 \\
(19.0)\end{array}$ & .057 & $\begin{array}{l}0.1 \\
(12.5)\end{array}$ & .78 \\
\hline $\begin{array}{l}\text { Emotional } \\
\text { Functioning }\end{array}$ & PM & 57 & $\begin{array}{l}73.2 \\
(17.2)\end{array}$ & 59 & $\begin{array}{l}75.6 \\
(15.1)\end{array}$ & PM & 39 & $\begin{array}{l}76.5 \\
(16.6)\end{array}$ & 42 & $\begin{array}{l}82.5 \\
(12.8)\end{array}$ & .26 & $\begin{array}{l}2.2 \\
(26.1)\end{array}$ & .69 & $\begin{array}{l}1.5 \\
(16.3)\end{array}$ & .93 \\
\hline Social Functioning & PM & 57 & $\begin{array}{l}85.9 \\
(19.2)\end{array}$ & 59 & $\begin{array}{l}91.0 \\
(11.9)\end{array}$ & PM & 39 & $\begin{array}{l}84.6 \\
(13.6)\end{array}$ & 42 & $\begin{array}{l}90.0 \\
(15.5)\end{array}$ & .84 & $\begin{array}{l}5.0 \\
(23.4)\end{array}$ & .16 & $\begin{array}{l}-1.1 \\
(13.9)\end{array}$ & .94 \\
\hline School Functioning & PM & 55 & $\begin{array}{l}80.1 \\
(17.1)\end{array}$ & 56 & $\begin{array}{l}88.7 \\
(12.2)\end{array}$ & PM & 39 & $\begin{array}{l}79.6 \\
(13.4)\end{array}$ & 42 & $\begin{array}{l}84.8 \\
(14.1)\end{array}$ & .43 & $\begin{array}{l}9.4 \\
(20.5)\end{array}$ & .002 & $\begin{array}{l}0.0 \\
(18.9)\end{array}$ & .41 \\
\hline \multicolumn{16}{|l|}{ PedsQL scales } \\
\hline Total Score & ICD & 13 & $\begin{array}{l}75.8 \\
(16.0)\end{array}$ & 13 & $\begin{array}{l}92.2 \\
(7.0)\end{array}$ & ICD & 12 & $\begin{array}{l}77.7 \\
(14.9)\end{array}$ & 13 & $\begin{array}{l}91.0 \\
(7.2)\end{array}$ & .16 & $\begin{array}{l}16.4 \\
(15.1)\end{array}$ & .009 & $\begin{array}{l}2.1 \\
(17.5)\end{array}$ & .41 \\
\hline $\begin{array}{l}\text { Physical Health } \\
\text { Summary }\end{array}$ & ICD & 13 & $\begin{array}{l}76.0 \\
(17.4)\end{array}$ & 13 & $\begin{array}{l}95.9 \\
(5.9)\end{array}$ & $I C D$ & 12 & $\begin{array}{l}76.0 \\
(19.8)\end{array}$ & 13 & $\begin{array}{l}90.1 \\
(9.4)\end{array}$ & .17 & $\begin{array}{l}20.0 \\
(16.5)\end{array}$ & .005 & $\begin{array}{l}-0.3 \\
(22.8)\end{array}$ & .53 \\
\hline $\begin{array}{l}\text { Psychosocial Health } \\
\text { Summary }\end{array}$ & ICD & 13 & $\begin{array}{l}75.6 \\
(16.8)\end{array}$ & 13 & $\begin{array}{l}88.6 \\
(11.3)\end{array}$ & $I C D$ & 12 & $\begin{array}{l}79.4 \\
(12.2)\end{array}$ & 13 & $\begin{array}{l}91.8 \\
(5.7)\end{array}$ & .75 & $\begin{array}{l}12.9 \\
(17.8)\end{array}$ & .02 & $\begin{array}{l}4.4 \\
(15.0)\end{array}$ & .33 \\
\hline $\begin{array}{l}\text { Emotional } \\
\text { Functioning }\end{array}$ & ICD & 13 & $\begin{array}{l}72.3 \\
(23.3)\end{array}$ & 13 & $\begin{array}{l}84.6 \\
(14.1)\end{array}$ & ICD & 12 & $\begin{array}{l}79.2 \\
(14.4)\end{array}$ & 13 & $\begin{array}{l}89.2 \\
(9.8)\end{array}$ & .62 & $\begin{array}{l}12.3 \\
(21.4)\end{array}$ & .06 & $\begin{array}{l}7.1 \\
(17.9)\end{array}$ & .21 \\
\hline Social Functioning & ICD & 13 & $\begin{array}{l}80.4 \\
(19.1)\end{array}$ & 13 & $\begin{array}{l}93.5 \\
(11.3)\end{array}$ & ICD & 12 & $\begin{array}{l}82.5 \\
(13.7)\end{array}$ & 13 & $\begin{array}{l}95.4 \\
(8.3)\end{array}$ & .79 & $\begin{array}{l}13.1 \\
(21.5)\end{array}$ & .06 & $\begin{array}{l}1.7 \\
(22.4)\end{array}$ & .39 \\
\hline School Functioning & ICD & 13 & $\begin{array}{l}74.2 \\
(18.0)\end{array}$ & 13 & $\begin{array}{l}87.7 \\
(13.0)\end{array}$ & ICD & 12 & $\begin{array}{l}76.7 \\
(15.1)\end{array}$ & 13 & $\begin{array}{l}90.8 \\
(7.9)\end{array}$ & .88 & $\begin{array}{l}13.5 \\
(21.4)\end{array}$ & .06 & $\begin{array}{l}4.6 \\
(18.6)\end{array}$ & .73 \\
\hline
\end{tabular}

Higher scores indicate better health-related quality of life

PedsQL Pediatric quality of life inventory, PM Pacemaker, ICD Implantable cardioverter defibrillator

${ }^{+}$Calculation for the interaction: Let $Y_{P P r}, Y_{C P r}, Y_{P S}, Y_{C S}$ define HRQoL of the patients and controls for self- and proxy reports, respectively. Then the difference ( $Y_{P P r}{ }^{-}$ $\left.\mathrm{Y}_{\mathrm{CPr}}\right)-\left(\mathrm{Y}_{\mathrm{PS}} \mathrm{Y}_{\mathrm{CS}}\right)$ was calculated and tested against 0 by Wilcoxon test

Significant values are indicated in bold 


\section{PCQLI parent proxy-report form \\ PCQLI child self-report form}

Total sample

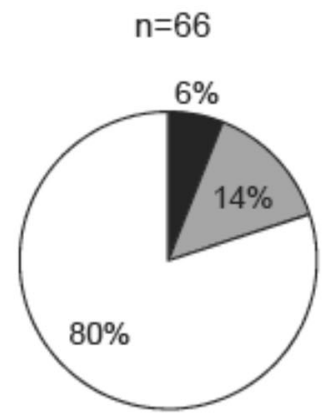

PM patients

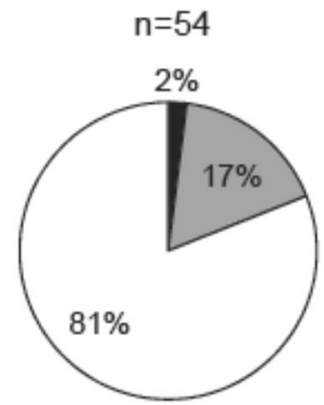

ICD patients

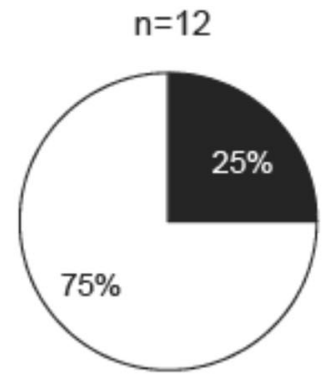

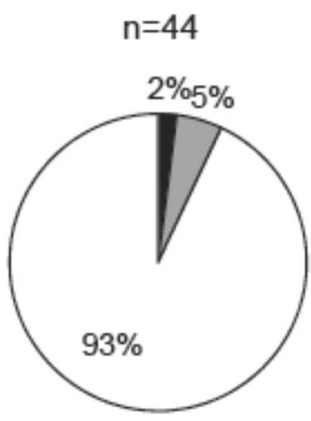

$\mathrm{n}=33$

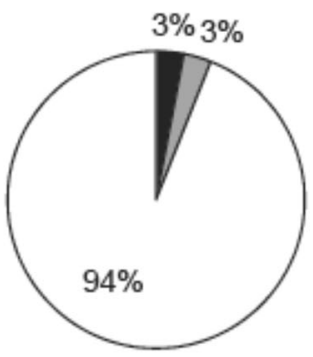

$n=11$

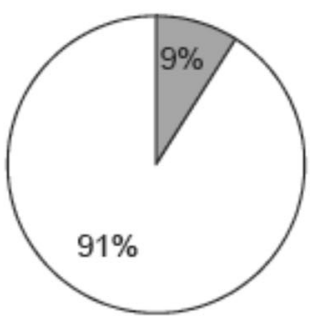

very low disease-specific HRQoL

low disease-specific HRQoL

normal disease-specific HRQoL

Fig. 1 Number of pacemaker (PM) and implantable cardioverter-defibrillator (ICD) patients with very low, low, and normal disease-specific healthrelated quality of life (HRQoL) assessed by the Pediatric Cardiac Quality of Life Inventory (PCQLI)

the presence of another non-cardiac chronic disease was associated with lower physical health. Disease-specific overall HRQoL was only marginally associated with child age at assessment, device type, and structural CHD $(p<.10)$. Younger patients, patients with ICD, and those with a structural CHD had lower disease-specific HRQoL than older patients, patients with PM, and those with no CHD.

\section{Discussion}

This study shows that pediatric PM and ICD patients have lower generic HRQoL than age- and sex-matched healthy controls, and that patients who need cardiac medication are seen as being at great risk for reduced generic HRQoL. The study also shows that the majority of the patients have normal proxy- and selfreported disease-specific HRQoL. Further, younger 
Table 3 Inter-correlations between total generic and disease-specific HRQOL scores (proxy-report form) and child sociodemographic and medical characteristics

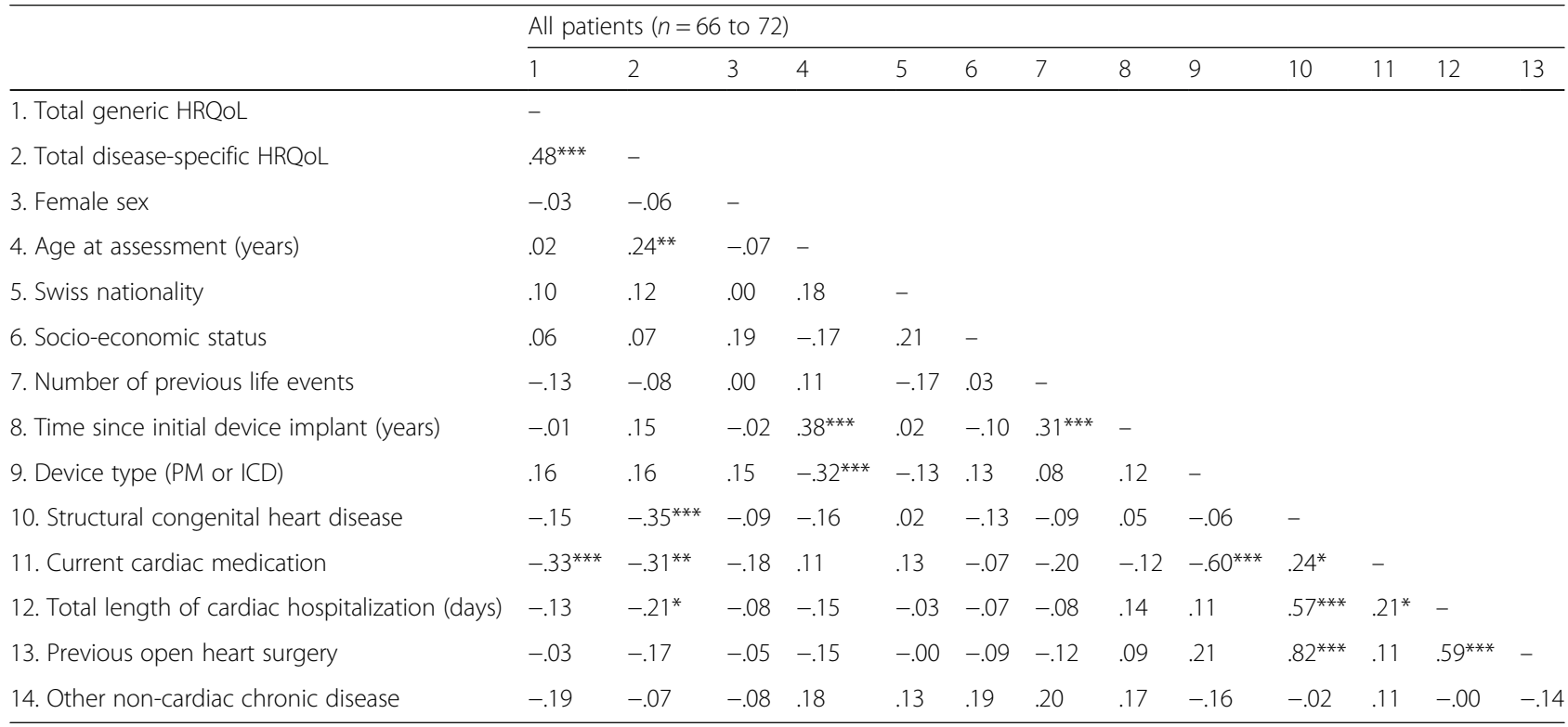

Kendall correlation coefficients are presented

Device type coded as $0=I C D, 1=\mathrm{PM}$; Structural congenital heart disease coded as $0=$ no, $1=$ yes; Current cardiac medication coded as $0=$ no, $1=$ yes; Previous open heart surgery coded as $0=$ no, $1=$ yes; Other chronic disease coded as $0=$ no, $1=$ yes (any other chronic disease than heart disease)

$H R Q o L$ Health-related quality of life

${ }^{*} p \leq .05,{ }^{* *} p \leq .01,{ }^{* * *} p \leq .001$

patients, patients with ICDs, and those with a structural CHD may have a higher risk for lower proxyreported disease-specific HRQoL.

In line with our hypothesis and with previous studies with PM patients, ICD patients, or both [7, 8, 10-13], pediatric PM and ICD patients showed lower self- and proxy-reported overall HRQoL and lower physical health than sex- and age-matched healthy controls. Lower psychosocial health was only found in ICD patients. In our study, healthy controls had higher SES and had experienced fewer previous life events than the patients. Consequently, we examined whether these variables might influence the HRQoL differences between patients and controls. A multiple linear regression with generic overall HRQoL differences as dependent variable showed no significant effect of SES or of number of previous life events on HRQoL differences between patients and controls (data not shown). A multitude of risk factors might influence the significant HRQoL differences between patients and controls (e.g. mental disorder of a parent, marital disharmony, lack of social support [25]). However, PM and ICD patients have to cope with at least one more life event than do healthy controls. The presence of a chronic disease with associated challenges such as arrhythmia-related symptoms, post-operative complications, device-related complications, and regular outpatient visits for clinical and device examination, which might be experienced as a threat exceeding the resources of the patients and their families [26] might be reflected in reduced HRQoL. Our study showed that parents of $20 \%$ of the patients and $7 \%$ of the patients themselves reported very low or low disease-specific HRQoL. Thus, some PM and ICD patients are at great risk for low HRQoL. Our data shows that parents and patients reported about the same level of generic HRQoL, while parents reported lower disease-specific HRQoL than the patients themselves. Thus, we may assume that the parents' subjective experience of the child's disease may especially bias their disease-specific proxy-reports.

In multivariate analyses, generic and disease-specific HRQoL were not predicted by the same variables. This probably reflects the scope of the questionnaires used. We decided to use the PedsQL 4.0 Generic Core scales [17] and the PCQLI $[18,19]$. While the PedsQL assesses the full range of health conditions (physical, social, emotional, and school functioning) and can be used to report HRQoL independently of the actual health state of the individual [27], the PCQLI refers to the unique challenges of a heart disease [27] and, thus, may more comprehensively report the challenges faced by PM and ICD patients [28]. This is also shown by the higher percentage of total variance explained by the predictors selected for disease-specific HRQoL. Our study showed that the need for antiarrhythmic or heart failure medication was associated with lower generic overall HRQoL and physical health. This finding is in line with the only previous study which analyzed the association between medication and HRQoL exclusively 
Table 4 Summary of multiple linear regression analyses predicting proxy-reported generic and disease-specific HRQoL of patients with $P M / I C D$

\begin{tabular}{|c|c|c|c|c|c|c|c|c|c|c|c|c|c|c|c|}
\hline \multirow{2}{*}{$\begin{array}{l}\text { Generic HRQoL } \\
\text { Predictor variable }\end{array}$} & \multicolumn{5}{|c|}{ Generic overall HRQoL $(n=70)$} & \multicolumn{5}{|c|}{ Physical Health Summary } & \multicolumn{5}{|c|}{ Psychosocial Health Summary } \\
\hline & B & SEB & $\beta$ & t & $p$ & B & SEB & $\beta$ & t & $p$ & B & SEB & $\beta$ & t & $p$ \\
\hline Female sex & -2.98 & 3.49 & -.10 & -.86 & .40 & -4.54 & 3.91 & -.13 & -1.16 & .25 & -1.43 & 3.59 & -.05 & -.40 & .69 \\
\hline Age at assessment (years) & .47 & .55 & .14 & .85 & .39 & .33 & .62 & .09 & .53 & .60 & .61 & .57 & .19 & 1.08 & .29 \\
\hline Time since initial device implant (years) & -.66 & .53 & -.19 & -1.25 & .22 & -.64 & .59 & -.17 & -1.08 & .28 & -.67 & .54 & -.20 & -1.24 & .22 \\
\hline Device type (PM or ICD) & 2.12 & 6.35 & .06 & .33 & .74 & 2.01 & 7.11 & .05 & .28 & .78 & 2.23 & 6.53 & .06 & .34 & .73 \\
\hline Structural congenital heart disease & 2.69 & 4.10 & .09 & .66 & .51 & 3.46 & 4.59 & .10 & .75 & .45 & 1.93 & 4.21 & .07 & .46 & .65 \\
\hline Current cardiac medication & -12.73 & 5.14 & -.39 & -2.48 & .02 & -16.45 & 5.75 & -.44 & -2.86 & .006 & -9.02 & 5.28 & -.28 & -1.71 & .09 \\
\hline Total length of cardiac hospitalization (days) & -.05 & .04 & -.18 & -1.27 & .21 & -.04 & .04 & -.13 & -.93 & .36 & -.06 & .04 & -.22 & -1.45 & .15 \\
\hline \multirow[t]{2}{*}{ Other non-cardiac chronic disease } & -8.69 & 4.50 & -.22 & -1.93 & .06 & -10.20 & 5.04 & -.23 & -2.03 & .05 & -7.19 & 4.62 & -.19 & -1.56 & .13 \\
\hline & \multicolumn{5}{|c|}{$\begin{array}{l}F=2.78, p=.01, R^{2}=.27 \\
R^{2} \text { adjusted }=.17\end{array}$} & \multicolumn{5}{|c|}{$\begin{array}{l}F=3.11, p=.005, R^{2}=.29 \\
R^{2} \text { adjusted }=.20\end{array}$} & \multicolumn{5}{|c|}{$\begin{array}{l}F=1.93, p=.07, R^{2}=.20 \\
R^{2} \text { adjusted }=.10\end{array}$} \\
\hline Disease-specific HRQoL & \multicolumn{5}{|c|}{$\begin{array}{l}\text { Disease-specific overall HRQoL } \\
(n=66)\end{array}$} & & & & & & & & & & \\
\hline Predictor variable & B & SEB & $\beta$ & t & $p$ & & & & & & & & & & \\
\hline Female sex & -4.14 & 3.54 & -.13 & -1.17 & .25 & & & & & & & & & & \\
\hline Age at assessment (years) & 1.06 & .55 & .31 & 1.91 & .06 & & & & & & & & & & \\
\hline Time since initial device implant (years) & .06 & .53 & .02 & .10 & .92 & & & & & & & & & & \\
\hline Device type (PM or ICD) & 12.03 & 6.43 & .29 & 1.87 & .07 & & & & & & & & & & \\
\hline Structural congenital heart disease & -7.72 & 4.21 & -.24 & -1.83 & .07 & & & & & & & & & & \\
\hline Current cardiac medication & -4.50 & 5.10 & -.13 & -.88 & .38 & & & & & & & & & & \\
\hline Total length of cardiac hospitalization (days) & -.04 & .04 & -.14 & -.98 & .33 & & & & & & & & & & \\
\hline \multirow[t]{2}{*}{ Other non-cardiac chronic disease } & -5.88 & 4.50 & -.14 & -1.31 & .20 & & & & & & & & & & \\
\hline & \multicolumn{5}{|c|}{$\begin{array}{l}F=4.17, p \leq .001, R^{2}=.37, \\
R^{2} \text { adjusted }=.28\end{array}$} & & & & & & & & & & \\
\hline
\end{tabular}

Device type coded as $0=I C D, 1=P M$; Structural congenital heart disease coded as $0=$ no, $1=$ yes; Current cardiac medication coded as $0=$ no, $1=$ yes; Other chronic disease coded as $0=$ no, $1=$ yes (any other chronic disease than heart disease)

$B$ Regression coefficient (unstandardized), SEB Standard error of the regression coefficient, $\beta$ Standardized regression coefficient

Significant values are indicated in bold

in PM patients [15]. Thus, pediatric PM and ICD patients who take medication are seen as being at risk for lower generic HRQoL by their parents. Three hypotheses are close: First, it could be assumed that these patients have greater disease-related problems than patients without medication. Second, it might be possible that these patients already had a lower HRQoL before the onset of their arrhythmia and that patients with low HRQoL are more likely to be prescribed medication. Third, it might be that the intake of medication reminds the patients and their parents of the disease, which can be reflected in lower HRQoL scores. However, in the context of this study, we were not able to prove these hypotheses and whether medication is an intermediating factor or not. Future studies might address this. Disease-specific HRQoL was only marginally predicted by child age, device type, and structural CHD. Younger patients, patients with ICDs, and those with CHD tended to have lower disease-specific HRQoL than older patients, patients with PM, and those without CHD. This result is in line with a previous study's finding that patients with ICD and a structural CHD have lower HRQoL [7].

The strengths of the current study include the use of standardized and well-validated HRQoL instruments and the presentation of data on both generic and diseasespecific HRQoL, which are seen as essential complements to each other [23] and which allow the influence of medical risk factors on each to be compared. Nevertheless, several limitations merit note. First, even though we achieved a response rate of $80 \%$ and our analyses revealed no significant differences between study participants and non-participants in sex, age at study beginning, device type, age at initial device implantation, presence of structural CHD, or the total length of cardiac hospitalization, we cannot exclude the possibility that the patients who participated in our study differed with respect to other variables (e.g. HRQoL) from those who did not. In addition, the possibility that some crosscontamination occurred between patients and controls 
cannot be excluded, since $40 \%$ of controls were recruited via patients' best friends. Second, the cross-sectional design of our study prevents us from drawing any conclusion about causal relations, and we are not able to describe the course of HRQoL over time. Third, our study included a rather small number of ICD patients, making it difficult to compare both device groups with respect to socio-demographic and medical characteristics. In terms of a post-hoc power analysis $(\alpha=.05$, twotailed) using $G^{*}$ power software [29], our sample size provided sufficient power to detect moderate effect sizes within the multiple regression analysis predicting proxyreported generic and disease-specific HRQoL. However, we were not able to predict self-reported generic and disease-specific HRQoL. Fourth, our study shows that socio-demographic and medical factors explain only a relatively small proportion of the patients' HRQoL outcome variance. This seems to imply that other factors not assessed in the current study influence the patients' HRQoL. In fact, we were unable to provide data on patients' social support or parental well-being or to define its proportion of variance explaining either generic or disease-specific HRQoL.

\section{Conclusions}

Our study shows that PM and ICD patients have lower generic overall HRQoL compared to healthy controls and that patients who need cardiac medication are at great risk for lower generic HRQoL. Furthermore, our study indicated a trend towards higher risk for low disease-specific HRQoL in younger patients, ICD patients, and patients with a structural CHD. Special attention should be given to these patients. They may benefit from a timely clinical evaluation of their HRQoL in order to provide supportive interventions.

\section{Abbreviations \\ HRQoL: Health-related quality of life; ICD: Implantable cardioverter- defibrillators; PCQLI: Pediatric Cardiac Quality of Life Inventory; PM: Pacemakers; SES: SocioEconomic Status}

\section{Acknowledgments}

We thank all children and their families for participating in the study. Dr. Luciano Molinari for statistical advice and Dr. Simon Milligan for language editing of our manuscript.

\section{Authors' contributions}

HW made substantial contributions to the study, including conceptualization, design, methodology, data curation, and formal analysis, and she wrote and revised the manuscript. PL and CB made substantial contributions to the study, including conceptualization, design, methodology, and data curation, and they reviewed and edited the manuscript. AR, KS and SH made substantial contributions to the study, including methodology and data curation, and reviewed/edited the manuscript. All authors read and approved the final version of the manuscript.

\section{Funding}

This study did not receive any grant from funding agencies in the public, commercial, or not-for-profit organization.

\section{Availability of data and materials}

The datasets used and analyzed during the current study are available from the corresponding author on reasonable request.

\section{Ethics approval and consent to participate}

This study was approved by the Ethical Review Board of the Canton of Zurich, Switzerland, and was performed in full accordance with the Declaration of Helsinki (2015-0322). All parents provided written informed consent after study procedures and aims had been explained in detail. All children provided verbal assent, while adolescents older than 14 years of age also provided written informed consent.

\section{Consent for publication}

Not applicable.

\section{Competing interests}

The authors declare that they have no competing interests.

\section{Author details}

'Department of Psychosomatics and Psychiatry, University Children's Hospital, Steinwiesstrasse 75, CH-8032 Zurich, Switzerland. ${ }^{2}$ Division of Child and Adolescent Health, Institute of Psychology, University of Zurich, Binzmühlestrasse 14, Box 8, CH-8050 Zürich, Switzerland. ${ }^{3}$ Children's Research Center, University Children's Hospital, Steinwiesstrasse 75, CH-8032 Zurich, Switzerland. ${ }^{4}$ Department of Cardiology, Pediatric Heart Centre, University Children's Hospital, Steinwiesstrasse 75, CH-8032 Zurich, Switzerland.

Received: 27 June 2018 Accepted: 6 September 2019

Published online: 11 October 2019

\section{References}

1. Taylor S, Zeigler V, Clark J. In: Zeigler V, Gilette P, editors. Permanent pacemakers. Armonk: Futura Publishing Company; 2001.

2. Koenig P. In: Koenig P, Hijazi Z, Zimmerman F, editors. Essentials of pediatric cardiology. New York: McGraw-Hill Medical Publishing Division; 2004.

3. Epstein AEDJ, Ellenbogen KA, Estes NA 3rd, Freedman RA, Gettes LS, Gillinov AM, Gregoratos G, Hammill SC, Hayes DL, Hlatky MA, Newby LK, Page RL, Schoenfeld MH, Silka MJ, Stevenson LW, Sweeney MO, Tracy CM, Epstein AE, Darbar D, DiMarco JP, Dunbar SB, Estes NA 3rd, Ferguson TB Jr, Hammill SC, Karasik PE, Link MS, Marine JE, Schoenfeld MH, Shanker AJ, Silka MJ, Stevenson LW, Stevenson WG, Varosy PD, American College of Cardiology Foundation, American Heart Association Task Force on Practice Guidelines, Heart Rhythm Society. 2012 ACCF/ AHA/HRS focused update incorporated into the ACCF/AHA/HRS 2008 guidelines for device-based therapy of cardiac rhythm abnormalities: a report of the American College of Cardiology Foundation/American Heart Association Task Force on Practice Guidelines and the Heart Rhythm Society. J Am Coll Cardiol. 2013;61(3):e6-75.

4. Keane J, Lock J, Fyler D. Nadas' pediatric cardiology. Keane JN, AS, editor. Philadelphia: Saunders; 2006.

5. Alpern D, Uzark K, Dick M. Psychosocial responses of children to cardiac pacemakers. J Pediatr. 1989;114(3):494-501.

6. Leidy NK, Revicki DA, Genesté B. Recommendations for evaluating the validity of quality of life claims for labeling and promotion. Value Health. 1999;2(2):113-27.

7. Czosek RJ, Bonney WJ, Cassedy A, Mah DY, Tanel RE, Imundo JR, et al. Impact of cardiac devices on the quality of life in pediatric patients. Circ Arrhythm Electrophysiol. 2012;5(6):1064-72.

8. Sears SF, Hazelton AG, St Amant J, Matchett M, Kovacs A, Vazquez LD, et al. Quality of life in pediatric patients with implantable cardioverter defibrillators. Am J Cardiol. 2011;107(7):1023-7.

9. Gaies MG, Watnick CS, Gurney JG, Bove EL, Goldberg CS. Health-related quality of life in patients with congenitally corrected transposition of the great arteries. J Thorac Cardiov Sur. 2011;142(1):136-41.

10. Cheng P, Gutierrez-Colina AM, Loiselle KA, Strieper M, Frias P, Gooden K, et al. Health related quality of life and social support in pediatric patients with pacemakers. J Clin Psychol Med Settings. 2014;21(1):92-102.

11. Costa R, da Silva KR, Mendonca RC, Nishioka S, Siqueira S, Tamaki WT, et al. Incidence of shock and quality of life in young patients with implantable cardioverter-defibrillator. Arq Bras Cardiol. 2007;88(3):258-64. 
12. DeMaso DR, Lauretti A, Spieth L, van der Feen JR, Jay KS, Gauvreau K, et al. Psychosocial factors and quality of life in children and adolescents with implantable cardioverter-defibrillators. Am J Cardiol. 2004;93(5):582-7.

13. Webster G, Panek KA, Labella M, Taylor GA, Gauvreau K, Cecchin F, et al. Psychiatric functioning and quality of life in young patients with cardiac rhythm devices. Pediatrics. 2014;133(4):e964-72.

14. Czosek RJ, Cassedy AE, Wray J, Wernovsky G, Newburger JW, Mussatto KA, et al. Quality of life in pediatric patients affected by electrophysiologic disease. Heart Rhythm. 2015;12(5):899-908.

15. da Silva KR, Costa R, De Oliveira RM, Lacerda MS, Huang AIU, Rossi MB, et al. Quality of life and functional capacity after long-term right ventricular pacing in pediatrics and young adults with congenital atrioventricular block. Pace. 2013;36(12):1539-49.

16. Priori SG, Blomstrom-Lundqvist C, Mazzanti A, Blom N, Borggrefe M, Camm J, et al. 2015 ESC guidelines for the management of patients with ventricular arrhythmias and the prevention of sudden cardiac death: the task force for the management of patients with ventricular arrhythmias and the prevention of sudden cardiac death of the European Society of Cardiology (ESC). Endorsed by: Association for European Paediatric and Congenital Cardiology (AEPC). Eur Heart J. 2015;36(41):2793-867.

17. Varni JW, Seid M, Kurtin PS. PedsQL 4.0: reliability and validity of the pediatric quality of life inventory version 4.0 generic core scales in healthy and patient populations. Med Care. 2001;39(8):800-12.

18. Goldbeck L, Borth-Bruns T. Translating, validation, and implementation of an instrument to assess health-related quality of life for children and adolescents with a chronic heart disease (project completion report). University U, editor. Ulm: Working Group: Behavioural Medicine; 2013.

19. Niemitz M, Seitz DC, Oebels M, Schranz D, Hovels-Gurich H, Hofbeck M, et al. The development and validation of a health-related quality of life questionnaire for pre-school children with a chronic heart disease. Qual Life Res. 2013;22(10):2877-88.

20. Goldbeck L, Seitz D, Niemitz M. PCQLI Inventar zur Erfassung der Lebensqualität herzkranker Vorschulkinder, Kinder und Jugendlicher: Manual. Ulm: Universität Ulm; 2012.

21. Landolt MA, Vollrath M. Life event scale. Manuscript U, editor. Zurich: University Childern's Hospital Zurich; 1998.

22. Largo RH, Pfister D, Molinari L, Kundu S, Lipp A, Duc G. Significance of prenatal, perinatal and postnatal factors in the development of AGA preterm infants at five to seven years. Dev Med Child Neurol. 1989; 31(4):440-56.

23. Matza LS, Swensen AR, Flood EM, Secnik K, Leidy NK. Assessment of healthrelated quality of life in children: a review of conceptual, methodological, and regulatory issues. Value Health. 2004;7(1):79-92.

24. Field A. Discovering statistics using SPSS. Edition T, editor. London: Sage Publications; 2009.

25. Ihle W, Frenzel T, Esser G. Entwicklungspsychopathologie und Entwicklungsepidemiologie. In: Esser G, editor. Lehrbuch der Klinischen Psychologie und Psychotherapie bei Kindern und Jugendlichen. Stuttgart: Georg Thieme Verlag; 2011.

26. Lazarus RS. Psychological stress and the coping process. New York: McGrawHill; 1966.

27. Bullinger M, Quitmann J. Quality of life as patient-reported outcomes: principles of assessment. Dialogues Clin Neurosci. 2014;16(2):137-45.

28. Marino E, Grey ST. A new role for an old player: do B cells unleash the self-reactive CD8+ $T$ cell storm necessary for the development of type 1 diabetes? J Autoimmun. 2008:31(3):301-5.

29. Faul F, Erdfelder E, Lang AG, Buchner A. G*Power 3: a flexible statistical power analysis program for the social, behavioral, and biomedical sciences. Behav Res Methods. 2007;39(2):175-91.

\section{Publisher's Note}

Springer Nature remains neutral with regard to jurisdictional claims in published maps and institutional affiliations.

Ready to submit your research? Choose BMC and benefit from:

- fast, convenient online submission

- thorough peer review by experienced researchers in your field

- rapid publication on acceptance

- support for research data, including large and complex data types

- gold Open Access which fosters wider collaboration and increased citations

- maximum visibility for your research: over $100 \mathrm{M}$ website views per year

At BMC, research is always in progress.

Learn more biomedcentral.com/submissions 